\title{
Nonlinear effective properties of unsaturated porous materials
}

\author{
Moran Wang ${ }^{1,2 *}$, Qun Chen ${ }^{3,4}$, Qinjun Kang ${ }^{1}$, Ning Pan ${ }^{4,5}$, Eli Ben-Naim ${ }^{2}$
}

\author{
1 Earth and Environmental Sciences Division, Los Alamos National Laboratory, Los Alamos, \\ NM 87545, USA \\ 2 Physics of Condensed Matter and Complex Systems Group and Center of Nonlinear Study, \\ Los Alamos National Laboratory, Los Alamos, NM 87545, USA \\ 3 Department of Engineering Mechanics, Tsinghua University, Beijing 100084, China \\ 4 Department of Biological and Agricultural Engineering, University of California, Davis, \\ CA 95616, USA \\ 5 Center of Physics of Fibrous Soft Matters, Donghua University, Shanghai 200051, China
}

\begin{abstract}
We investigate the nonlinear responses of effective properties of unsaturated porous materials using a numerical framework. The multiphase microstructure is reconstructed through a random generation-growth method, and the transport governing equations are solved efficiently by a lattice Boltzmann model. After validation, the present framework is used to study the nonlinear behavior of thermal conductivity and electrical permittivity caused by the saturation degree and the phase interaction for multiphase materials. The results show that the effective thermal conductivity of unsaturated porous materials increase and then decrease with the phase interaction ratio, while the effective permittivity decrease monotonously with the phase interaction. Mechanism analyses indicate that these nonlinear behavior lies in the role of the liquid phase in the transport. For the thermal conductivity, the liquid phase plays a "bridge" role because its conductivity is between those of the solid and the gas. A better bridge network would enhance the overall effective thermal conductivity. However for the electrical permittivity, the liquid phase plays the leading actor of transport. A better liquid phase connection will lead to a higher effective electrical permittivity of the multiphase system.
\end{abstract}

Keywords: effective property; unsaturated porous media; phase interaction; nonlinear response

\section{Introduction}

As well known, the effective properties of multiphase materials, such as the thermal conductivity and electric permittivity, depend on not only the properties and the volume fraction of each inclusion, but also the geometrical microstructures and the phase interaction effects between the components[1]. Predictions of effective properties of multiphase materials has been paid much attentions in the past fifty years because of their important applications in material design, geophysical exploration, biological and medical engineering[1-4]. Most

\footnotetext{
*Corresponding author. Email: mwang@lanl.gov
}

popular theoretical models for the effective properties of multiphase materials are based on networks of Parallel and Series modes, or introducing the morphology effects by some empirical parameters[5-7]. No analytical models have been found that deal successfully with effective properties of multiphase materials with random geometrical microstructures and phase interactions up to now. A complete numerical determination of the effective properties of multiphase media has to include such two major steps as first to reproduce properly the multiphase microstructures using computer algorithms, and then to solve the relevant transport governing equations with acceptable 
efficiency and accuracy.

Several methods have been proposed to reconstruct the microstructures of two-phase materials using computers[2, 3]. Moreover, for multiphase materials, the microstructures are influenced by the phase interactions. To our best knowledge, this issue has seldom been systematically investigated. A few researchers used the liquid-bridge structures for water distribution in unsaturated porous media $[8,9]$. Mohanty [10] adopted a Monte Carlo annealing algorithm to generate unsaturated porous media. Wang et al. [11-14] proposed a multi-parameter random generation-growth method to replicate random structures and investigated thermal properties of porous media.

The conventional partial differential equation (PDE) solvers have been applied to tackle the governing equations in multiphase media of multiphysical transports [15-17]. However the structural complexities bring in two difficulties: the requirement of grid refinements for complex structures and the conjugate constraints at interfaces between phases, both of which bring the calculations using any convectional PDE solver to an unacceptably expensive level. Owing to its easy implementation of multiple inter-particle interactions and complex geometry boundary conditions [18-21], and that in general the conservation laws can hold automatically without additional computational efforts [22, 23], the lattice Boltzmann method (LBM) has recently been developed successfully for modeling of hydrodynamics [19, 21, 22], and other transport process [23-28] in multiphase systems and the effective properties can be consequently calculated.

This work is aiming to reveal the nonlinear response of effective properties of unsaturated porous materials using a numerical framework. The rest of this paper is arranged as follows. Section 2 introduces the essential numerical framework for this study. After validations, the present numerical tool is employed to predict the effective properties of unsaturated porous materials in Section 3. The mechanism will be discussed based on our results.

\section{Numerical framework}

\subsection{Structure reproduction}

Here we use our random generation-growth method to reproduce the random microstructure of multiphase materials. The algorithm is described as follows.[12]

I) Randomly locate the cores of the first growing phase in a grid system based on a seed distribution probability, $C_{d}$. Each cell in the grid will be assigned a random number by a uniform distribution function within $(0,1)$, whose random number is no greater than $C_{d}$ will be chosen as a seed; II) Expand every element of the growing phase to its neighboring cells in all directions based on each given directional growth probability, $D_{i}$, where $i$ represents the direction. Again for each growing element, new random numbers will be assigned to its neighboring cells. The neighboring cell in direction $i$ will become part of the growing phase if its random number is no greater than $D_{i}$, III) Repeat the growing process of II) until the volume fraction of the first growing phase reaches its given value $\Phi^{2}$; IV) For multiphase materials, the $n$-th phase ( $n>2)$ will grow based on a phase interaction growth probability, $\mathrm{I}_{\mathrm{i}}^{\mathrm{n}, \mathrm{m}}$, which represents the growth probability of the $\mathrm{n}$-th phase on the m-th phase along the $i$-th direction; V) Stop the $\mathrm{n}$-th phase growth once its volume fraction reaches the given value $\Phi^{n}$; VI) Repeat the next phase growth as described in IV) $\&$ V) until $n=\mathrm{N}$; VII) The spaces not occupied at the end represent the non-growing phase.

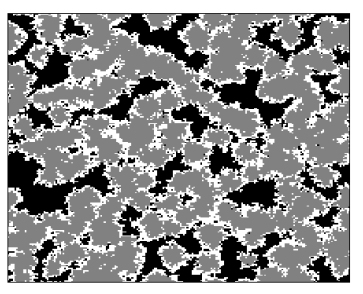

(a) $I_{i}^{l, l}: I_{i}^{l, s}=1: 1$

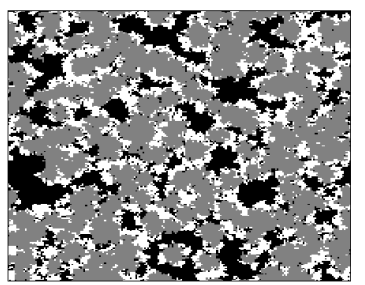

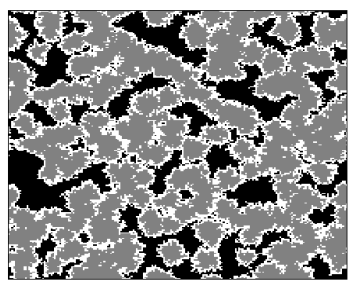

(b) $I_{i}^{l, l}: I_{i}^{l, s}=1: 10$

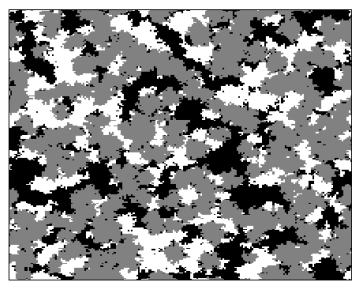



(c) $I_{i}^{l, l}: I_{i}^{l, s}=10: 1$
(d) $I_{i}^{l, l}: I_{i}^{l, s}=100: 1$

Fig. 1 Microstructures of 3-phase porous media with different phase interaction growth probabilities. The gray is the solid particles, the white the liquid, and the dark the gas.

In this work we emphasize the phase interaction $\left(I_{i}^{n, m},\right)$ effects on the microstructure and the properties of multiphase materials. For a three-phase porous medium, the simplest case is to generate the liquid phase with $I_{i}^{l, l}: I_{i}^{l, s}=1$ where $l$ represents the liquid phase and $s$ the solid phase. This hypothesis is based on a strong wetting effect caused by a strong liquid-solid attractive potential, and will result in a uniform liquid film attached on the solid grains as shown in Fig. 1(a). The smaller is the $I_{i}^{l, l}: I_{i}^{l, s}$ ratio, the more uniform is the liquid film [see Fig. 1(b)]. Such structures can be found in some multi-components composite materials. However for the unsaturated sandstones or glass assembles, the water tends to be as droplets rather than as films on solid surfaces. We can also reproduce similar water distributions as those in glass assembles by enlarging the $I_{i}^{l, l}: I_{i}^{l, s}$ ratio, as shown in Figs. 1(c) and (d). Fig. 1 compares the water distributions in porous media at different phase interaction growth probabilities. A greater $I_{i}^{l, l}: I_{i}^{l, s}$ ratio corresponds to a weaker wetting interface and the liquid is more aggregative.

\subsection{Governing equations}

Consider the thermal or electric transport through a multiphase material system as shown in Fig. 2. For a steady and single transport process without contact resistance, radiation, phase change or other influences from sources, the governing equations can be summarized as follows.
The thermal or electric transport is governed by a Laplace equation [29]:

$$
\nabla \cdot\left(\lambda_{n} \nabla \Phi\right)=0
$$

where $\lambda_{n}$ is the local conductivity of the $n$-th phase, which could be thermal conductivity or dielectric permittivity, and correspondingly, $\Phi$ would be the thermal potential (temperature) $[15$, $16]$ or the electric potential [17].

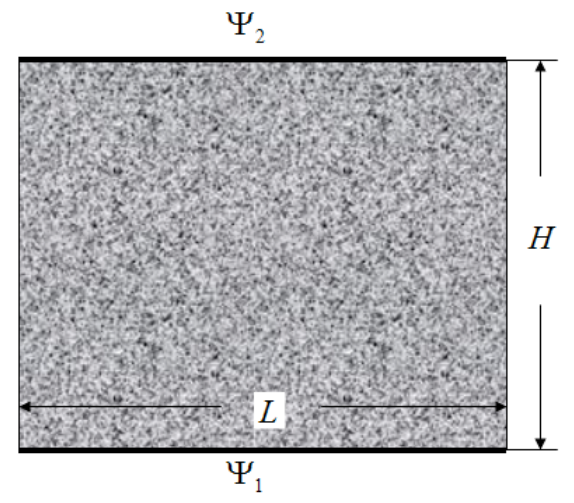

Fig. 2 Schematic diagram of domain and boundaries of a multiphase system

The boundary conditions are as follows.

$$
\begin{aligned}
& \left.\Psi\right|_{y=0}=\Psi_{1}, \\
& \left.\Psi\right|_{y=H}=\Psi_{2} .
\end{aligned}
$$

For the insulated boundaries on the sides, a specular reflection treatment is implemented to avoid "energy leak" along the surfaces[12]. At the interfaces between two phases ( $i$ and $j$ ) in equilibrium, the continuities of both potential and flux have to be satisfied, i.e.

$$
\begin{gathered}
\Phi_{\mathrm{int}, i}=\Phi_{\mathrm{int}, j} \\
\left.\lambda_{i} \nabla \Phi\right|_{\mathrm{int}, i}=\left.\lambda_{j} \nabla \Phi\right|_{\mathrm{int}, j},
\end{gathered}
$$

where the subscript "int" corresponds to the interfaces between phase "i" and " $\mathrm{j}$ ". Equation (1) governs the distribution of potential $\Phi$ in 
the multiphase material, subject to the interface constraints (2)-(5). The effective conductivity can be determined as

$$
\lambda_{e f f}=q H / \Delta \Phi
$$

where $q$ is the steady flux through the material between the potential difference $(\Delta \Phi)$ over a thickness $H$.

\subsection{Lattice Boltzmann solver}

The lattice Boltzmann method (LBM) is intrinsically a mesoscopic computational fluid dynamics (CFD) approach and has achieved considerable success in simulating fluid flows and associated transport [22]. The most important advantages of LBM include the easy implementations of multiple inter-particle interactions and complex geometry boundaries [18-21]. Conservations can generally hold automatically without additional computational efforts [23, 25].

For the governing equation, Eq. (1), we employ the evolution equation on discrete lattices for each phase as

$$
\begin{array}{r}
g_{\alpha}\left(\mathbf{r}+\mathbf{e}_{\alpha} \delta_{t}, t+\delta_{t}\right)-g_{\alpha}(\mathbf{r}, t)= \\
-\frac{1}{\tau^{n}}\left[g_{\alpha}(\mathbf{r}, t)-g_{\alpha}^{e q}(\mathbf{r}, t)\right],
\end{array}
$$

The equilibrium distribution of the evolution variable, $g_{\alpha}$, for the two-dimensional nine-speed (D2Q9) model is

$$
g_{\alpha}^{e q}=\left\{\begin{array}{lc}
0 & \alpha=0 \\
\Psi / 6 & \alpha=1,2,3,4 \\
\Psi / 12 & \alpha=5,6,7,8
\end{array},\right.
$$

and the dimensionless relaxation time

$$
\tau^{n}=\frac{3}{2} \frac{\lambda^{n}}{c^{2} \delta_{t}}+0.5
$$

where the subscript $n$ still represents the $n$-th phase, $\delta_{t}$ the time step, and $c$ a pseudo lattice speed whose value can theoretically take any positive value to insure $\tau$ values within (0.5, $2)[12,19]$. The potential and flux on each lattice are calculated by

$$
\begin{gathered}
\Psi=\sum_{\alpha} g_{\alpha} \\
q=\left(\sum_{\alpha} \mathbf{e}_{\alpha} g_{\alpha}\right) \frac{\tau^{n}-0.5}{\tau^{n}}
\end{gathered}
$$

\section{Results and discussion}

\subsection{Validations by experimental data}

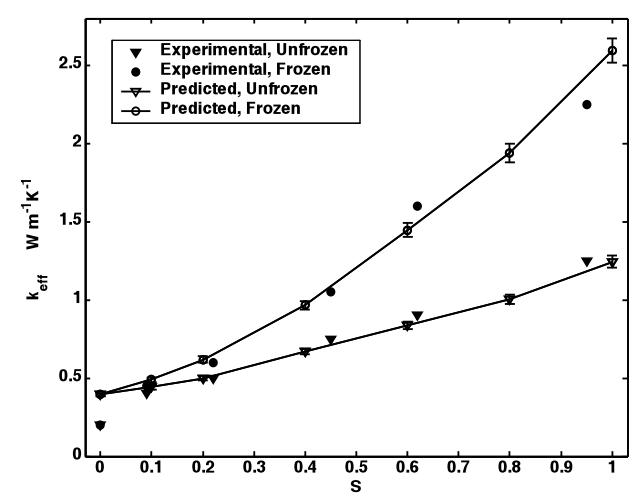

Fig. 3 Comparisons of the predicted thermal conductivities with the experimental data [30] for frozen and unfrozen soils.

Consider the thermal conductivity of an unsaturated soil. Customarily, the degree of saturation, $S$, is the liquid volume fraction in pores. The three-phase (3P) microstructure is reconstructed on a $200 \times 200$ grid with the parameters $C_{d}=0.01$, and $I_{i}^{w, w}=I_{i}^{w, s}$ for each $i$ direction. Fig. 3 shows the predicted thermal conductivities versus the degree of saturation at $\varepsilon=0.52$ for frozen and unfrozen sands. The thermal conductivities used in the simulations are $k_{s}=2.85 \mathrm{~W} / \mathrm{m} \cdot \mathrm{K}, \quad k_{w}=0.5924 \mathrm{~W} / \mathrm{m} \cdot \mathrm{K}, \quad k_{g}$ $=0.0249 \mathrm{~W} / \mathrm{m} \cdot \mathrm{K}$, and $k_{\text {ice }}=2.38 \mathrm{~W} / \mathrm{m} \cdot \mathrm{K}$ [30]. 
The numerical results are compared with the experimental data [30]. Good agreements are obtained with the experimental data for both frozen and unfrozen cases.

\subsection{Nonlinear responses of unsaturated porous materials}

The present numerical framework has been used to investigate the nonlinear responses of effective properties of unsaturated porous materials.

Fig. 4 shows the thermal conductivity of unsaturated soil as a function of water saturation for three different phase interaction ratios. The parameters are $\varepsilon=0.5, \quad k_{s}=2.85 \mathrm{~W} / \mathrm{m} \cdot \mathrm{K}, \quad k_{l}$ $=0.5924 \mathrm{~W} / \mathrm{m} \cdot \mathrm{K}$, and $k_{g}=0.0249 \mathrm{~W} / \mathrm{m} \cdot \mathrm{K}$. [30]. The results indicate that the effective thermal conductivity increases with the water content nonlinearly. The phase interaction ratio has a markable effect on the thermal conductivity when the saturation is between 0.2 and 0.6 ; otherwise, the phase interaction effect is negligible. Fig. 5 compares thermal conductivities for different phase interaction ratios when the saturation is given at 0.5 . When the phase interaction ratio varies from 0.01 to 300 , the effective thermal conductivity does not increase with the ratio monotonously. The thermal conductivity increase with the phase interaction ratio when $I<10$, and decrease with the phase interaction ratio when $I>100$. The effective thermal conductivity will reach a maximum when the phase interaction ratio is between 10 and 100 (around 50). Because the thermal conductivity of water is much higher than that of gas, the overall effective thermal conductivity of unsaturated porous materials will highly depend on the liquid bridges formed by the water. An appropriate value of the phase interaction ratio will form a good liquid-bridge network in the solid frame, rather than liquid films for low phase interaction ratios or liquid droplets for high ones, and therefore leads to a high effective thermal conductivity of the multiphase materials.

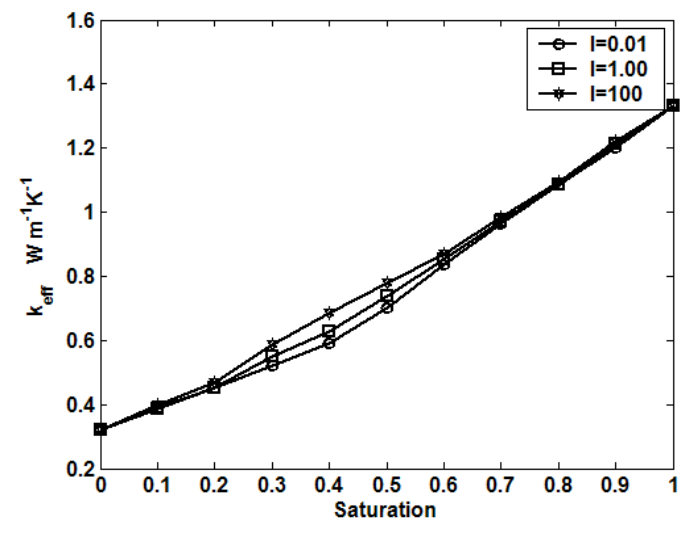

Fig. 4 Effective thermal conductivity vs. saturation for three different phase interaction ratios.

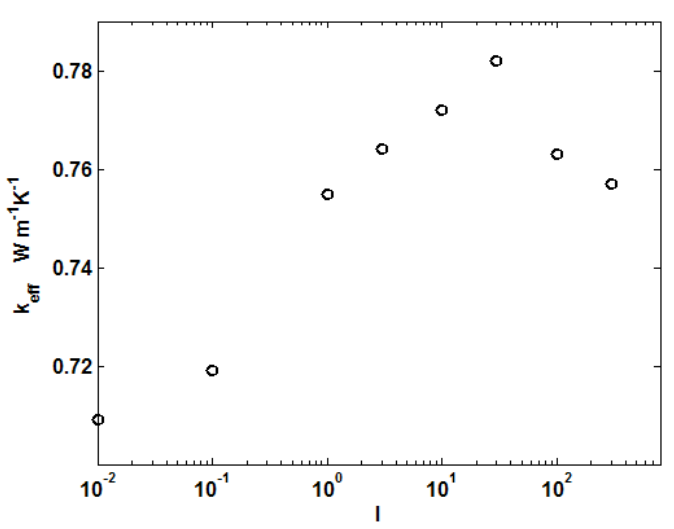

Fig. 5 Effective thermal conductivity as a function of phase interaction ratio.

When the present framework is applied to the electrical permittivity for the same unsaturated porous material system, the results are different, as shown in Figs. 6 and 7. The electrical permittivity for each component is: $\varepsilon_{\mathrm{r}}^{\mathrm{s}}=5.5, \quad \varepsilon_{\mathrm{r}}{ }^{1}=80$, and $\varepsilon_{\mathrm{r}}^{\mathrm{g}}=1$ [31]. Since the permittivity of liquid is much higher than both the other two phases, the phase interaction effect is much stronger compared with that in Fig. 5. The effect is only negligible when the saturation is smaller than 0.3 . 


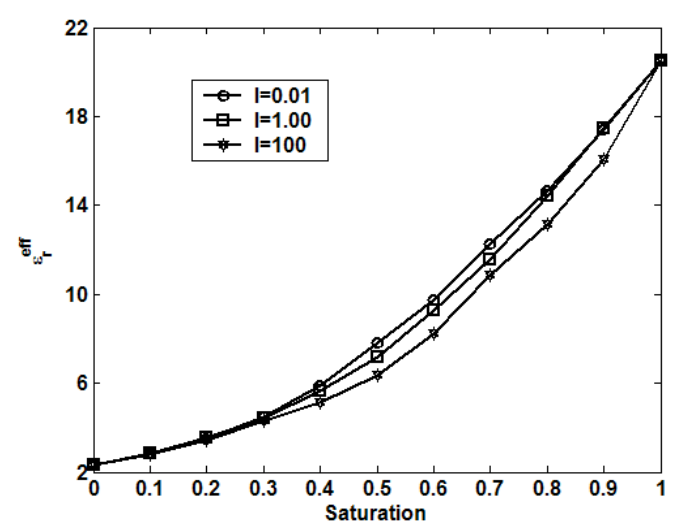

Fig. 6 Effective electrical permittivity vs. saturation for three different phase interaction ratios.

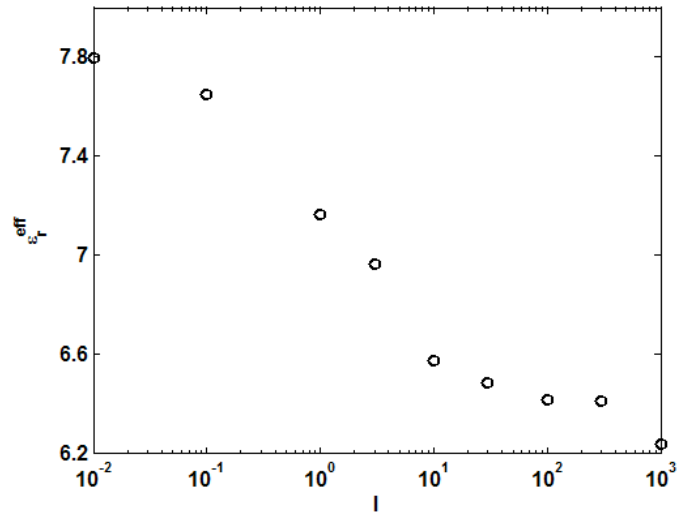

Fig. 7 Effective electrical permittivity as a function of phase interaction ratio.

Fig. 7 indicates that the effective electrical permittivity decreases sharply with the phase interaction ratio when the ratio is smaller than 10 , and then decreases smoothly for larger $I$ 's. This trend is quite different from that in Figure 6. The mechanism may lie in the different roles the liquid phase plays. For the thermal conductivity, the liquid phase plays a "bridge" role because its conductivity is between those of the solid and the gas. A better bridge network would enhance the overall thermal conductivity. However for the electrical permittivity, the liquid phase plays the leading actor of transport. The better the liquid phase connects itself, the higher the effective electrical permittivity for the multiphase system is.

\section{Conclusions}

The nonlinear responses of thermal conductivity and electrical permittivity caused by the saturation degree and the phase interaction have been studies for multiphase materials using a numerical framework. The results show that the effective thermal conductivity of unsaturated porous materials increase and then decrease with the phase interaction ratio, while the effective permittivity decrease monotonously with the phase interaction. The response of effective properties depends on the role of the liquid phase in the transport. For the thermal conductivity, the liquid phase plays a "bridge" role because its conductivity is between those of the solid and the gas. A better bridge network would enhance the overall effective thermal conductivity. However for the electrical permittivity, the liquid phase plays the leading actor of transport. The better the liquid phase connects itself, the higher the effective electrical permittivity for the multiphase system is.

\section{Reference}

[1] Ingham DB, Pop I. Transport Phenomena in Porous media III. Oxford, UK: Elsevier Science Pub. Co.; 2005.

[2] Li JCM, editor. Microstructure and Properties of Materials. Singapore: World Scientific; 1996.

[3] Torquato S. Random heterogeneous materials: microstructure and macroscopic properties. New York: Springer; 2002.

[4] Wang M, Pan N. Predictions of effective physical properties of complex multiphase materials. Mater Sci Eng R-Rep 2008 Dec;63(1):1-30.

[5] Liang $X G, Q u$ W. Effective thermal 
conductivity of gas-solid composite materials and the temperature difference effect at high temperature. International Journal of Heat and Mass Transfer 1999 May;42(10):1885-93.

[6] Friedman SP. A saturation degree-dependent composite spheres model for describing the effective dielectric constant of unsaturated porous media. Water Resources Research 1998;34(11):2949-61.

[7] Gao L, Gu JZ. Effective dielectric constant of a two-component material with shape distribution. Journal of Physics D-Applied Physics 2002;35(3):267-71.

[8] Geminard JC, Gayvallet H. Thermal conductivity of a partially wet granular material. Physical Review E 2001;64(4): 041301.

[9] Kohout M, Collier AP, Stepanek F. Effective thermal conductivity of wet particle assemblies. International Journal of Heat and Mass Transfer 2004;47(25):5565-74.

[10] Mohanty S. Effect of multiphase fluid saturation on the thermal conductivity of geologic media. Journal of Physics D-Applied Physics 1997;30(24):L80-L4.

[11] Wang MR, Pan N, Wang JK, Chen SY. Mesoscopic simulations of phase distribution effects on the effective thermal conductivity of microgranular porous media. Journal of Colloid and Interface Science 2007;311(2):562-70.

[12] Wang MR, Wang JK, Pan N, Chen SY. Mesoscopic predictions of the effective thermal conductivity for microscale random porous media. Physical Review E 2007;75(3):036702.

[13] Wang M, Pan N. Modeling and prediction of the effective thermal conductivity of random open-cell porous foams.
International Journal of Heat and Mass Transfer 2008 Mar;51(5-6):1325-31.

[14] Wang MR, He JH, Yu JY, Pan N. Lattice Boltzmann modeling of the effective thermal conductivity for fibrous materials. International Journal of Thermal Sciences 2007 Sep;46(9):848-55.

[15] Sutradhar A, Paulino GH. The simple boundary element method for transient heat conduction in functionally graded materials. Computer Methods in Applied Mechanics and Engineering 2004;193(42-44):4511-39.

[16] Zhang HF, Ge XS, Ye H. Effectiveness of the heat conduction reinforcement of particle filled composites. Modelling and Simulation in Materials Science and Engineering 2005;13(3):401-12.

[17] [17] Myroshnychenko V, Brosseau C. Finite-element method for calculation of the effective permittivity of random inhomogeneous media. Physical Review E 2005;71(1):016701.

[18] Wang M, Chen S. Electroosmosis in homogeneously charged micro- and nanoscale random porous media. Journal of Colloid and Interface Science 2007;314(1):264-73.

[19] Wang M, Pan N, Wang JK, Chen SY. Lattice Poisson-Boltzmann simulations of electroosmotic flows in charged anisotropic porous media. Communications in Computational Physics 2007; 2: 1055-70.

[20] Wang M, Wang JK, Chen SY, Pan N. Electrokinetic pumping effects of charged porous media in microchannels using the lattice Poisson-Boltzmann method. Journal of Colloid and Interface Science 2006; 304(1):246-53.

[21] Muhammed EK, Ahmet HA, Eyad M. Laboratory validation of lattice Boltzmann method for modeling pore-scale flow in 
granular materials. Computers and Geotechnics 2006;33(8):381-95.

[22] Chen S, Doolen GD. Lattice Boltzmann method for fluid flows. Annual Review of Fluid Mechanics 1998; 30:329-64.

[23] Wang J, Wang M, Li Z. Lattice evolution solution for the nonlinear Poisson-Boltzmann equation in confined domains. Communications in Nonlinear Science and Numerical Simulation 2008;13(3):575-83.

[24] He XY, Chen SY, Zhang RY. A lattice Boltzmann scheme for incompressible multiphase flow and its application in simulation of Rayleigh-Taylor instability. Journal of Computational Physics 1999;152(2):642-63.

[25] Wang JK, Wang M, Li ZX. A lattice Boltzmann algorithm for fluid-solid conjugate heat transfer. International Journal of Thermal Sciences 2007;46:228-34.

[26] Chen Q, Wang MR, Pan N, Guo ZY. Irreversibility of Heat Conduction in Complex Multiphase Systems and Its Application to the Effective Thermal Conductivity of Porous Media. Int J Nonlinear Sci Numer Simul 2009 Jan;10(1):57-66.

[27] He XY, Li N. Lattice Boltzmann simulation of electrochemical systems. Computer Physics Communications 2000;129(1-3):158-66.

[28] Wang MR, Kang QJ. Electrokinetic Transport in Microchannels with Random Roughness. Anal Chem 2009 Apr;81(8):2953-61.

[29] Tuncer E, Gubanski SM, Nettelblad B. Dielectric relaxation in dielectric mixtures: Application of the finite element method and its comparison with dielectric mixture formulas. Journal of Applied Physics
2001;89(12):8092-100.

[30] Singh AK, Singh R, Chaudhary DR. Prediction of Effective Thermal-Conductivity of Moist Porous Materials. Journal of Physics D-Applied Physics 1990;23(6):698-702.

[31] Miyamoto T, Annaka T, Chikushi J. Extended dual composite sphere model for determining dielectric permittivity of Andisols. Soil Science Society of America Journal 2005;69(1):23-9. 